\title{
Inequalities and Separation for a Biharmonic Laplace-Beltrami Differential Operator in a Hilbert Space Associated with the Existence and Uniqueness Theorem
}

\author{
ZAYED E. M. E.* \\ Department of Mathematics, Faculty of Science, Zagazig University, P.O. Box 44519, \\ Zagazig, Egypt.
}

Received 19 November 2015; Accepted 9 April 2016

Abstract. In this paper, we have studied the separation for the biharmonic LaplaceBeltrami differential operator

$$
A u(x)=-\Delta \Delta u(x)+V(x) u(x),
$$

for all $x \in R^{n}$, in the Hilbert space $H=L_{2}\left(R^{n}, H_{1}\right)$ with the operator potential $V(x) \in$ $C^{1}\left(R^{n}, L\left(H_{1}\right)\right)$, where $L\left(H_{1}\right)$ is the space of all bounded linear operators on the Hilbert space $H_{1}$, while $\Delta \Delta u$ is the biharmonic differential operator and

$$
\Delta u=-\sum_{i, j=1}^{n} \frac{1}{\sqrt{\operatorname{det} g}} \frac{\partial}{\partial x_{i}}\left[\sqrt{\operatorname{det} g} g^{-1}(x) \frac{\partial u}{\partial x_{j}}\right]
$$

is the Laplace-Beltrami differential operator in $R^{n}$. Here $g(x)=\left(g_{i j}(x)\right)$ is the Riemannian matrix, while $g^{-1}(x)$ is the inverse of the matrix $g(x)$. Moreover, we have studied the existence and uniqueness Theorem for the solution of the non-homogeneous biharmonic Laplace-Beltrami differential equation $A u=-\Delta \Delta u+V(x) u(x)=f(x)$ in the Hilbert space $H$ where $f(x) \in H$ as an application of the separation approach.

AMS Subject Classifications: 47F05, 58J99

Chinese Library Classifications: O175.29

Key Words: Separation biharmonic Laplace-Beltrami operator; operator potential; Hilbert space $L_{2}\left(R^{n}, H_{1}\right)$; coercive estimate; existence and uniqueness Theorem.

\section{Introduction}

The concept of separation for differential operators was first introduced by Everitt and Giertz [1,2]. They have obtained the separation results for the Sturm Liouville differential

${ }^{*}$ Corresponding author. Email address: e.m.e.zayed@hotmail.com (E. M. E. Zayed) 
operator

$$
A u(x)=-u^{\prime \prime}(x)+V(x) u(x), \quad x \in R,
$$

in the space $L_{2}(R)$. They have studied the following question: What are the conditions on $V(x)$ such that if $u(x) \in L_{2}(R)$ and $A u(x) \in L_{2}(R)$ imply that both of $u^{\prime \prime}(x)$ and $V(x) u(x) \in L_{2}(R)$ ? More fundamental results of separation for differential operators were obtained by Everitt and Giertz [3,4]. A number of results concerning the property referred to the separation of differential operators was discussed by Biomatov [5], Otelbaev [6], Zettle [7] and Mohamed et al. [8-13]. The separation for the differential operators with the matrix potential was first studied by Bergbaev [14]. Brown [15] has shown that certain properties of positive solutions of disconjugate second order differential expressions imply the separation. Some separation criteria and inequalities associated with linear second order differential operators have been studied by Brown et al. [16,17]. Mohamed et al. [11] have studied the separation property of the Sturm Liouville differential operator

$$
A u(x)=-\left(\mu(x) u^{\prime}\right)^{\prime}+V(x) u(x), \quad x \in R,
$$

in the Hilbert space $H_{p}(R),(p=1,2)$, where $V(x) \in L\left(l_{p}\right)$ is an operator potential which is a bounded linear operator on $l_{p}$ and $\mu(x) \in C^{1}(R)$ is a positive continuous function on $R$.

Mohamed et al. [9] have studied the separation property for the linear differential operator

$$
A u(x)=(-1)^{m} D^{2 m} u(x)+V(x) u(x), \quad x \in R,
$$

in the Banach space $L_{p}(R)^{l}$ where $V(x)$ is an $l \times l$ positive hermitian matrix and $D^{2 m}=$ $d^{2 m} / d x^{2 m}$ is the classical differentiation of order $2 m$.

Mohamed et al. [12] have studied the separation of the Schrodinger operator

$$
A u(x)=-\Delta u(x)+V(x) u(x), \quad x \in R^{n},
$$

with the operator potential $V(x) \in C^{1}\left(R^{n}, L\left(H_{1}\right)\right)$ in the Hilbert space $L_{2}\left(R^{n}, H_{1}\right)$ and $\Delta=$ $\sum_{i=1}^{n}\left(\partial^{2} / \partial x_{i}^{2}\right)$ is the Laplace operator in $R^{n}$.

Mohamed et al. [13] have studied the separation for the general second order differential operator

$$
A u(x)=-\sum_{i, j=1}^{n} \alpha_{i j}(x) D_{i}^{j} u(x)+V(x) u(x), \quad x \in R^{n},
$$

in the weighted Hilbert space $L_{2, k}\left(R^{n}, H_{1}\right)$ with a positive weight function $k(x)$ and the operator potential $V(x) \in C^{1}\left(R^{n}, L\left(H_{1}\right)\right)$ where $\alpha_{i j} \in C^{2}\left(R^{n}\right)$ and $D_{i}^{j}=\partial^{2} / \partial x_{i} \partial x_{j}$.

Zayed et al. [18] have obtained recent results on the separation of linear and nonlinear Schrodinger-type operators with the operator potentials in Banach spaces. Furthermore, 
Zayed et al. [19] have studied the separation of the elliptic differential operator

$$
A u(x)=-\sum_{i, j=1}^{n}\left[D_{i}\left(P_{i j}(x) D_{j} u(x)\right)-P_{i j}(x) b_{i}(x) b_{j}(x) u(x)\right]+V(x) u(x),
$$

for all $x \in R^{n}$, in the weighted Hilbert space $L_{2, k}\left(R^{n}, H_{1}\right)$ with the operator potential $V(x) \in$ $C^{1}\left(R^{n}, L\left(H_{1}\right)\right)$, where $P_{i j}(x)$ and $b_{i}(x)$ are real-valued continuous function, while $D_{i}=$ $\partial / \partial x_{i}$.

Zayed et al. [20] recently have studied the separation for the Laplace Beltrami differential operator

$$
A u(x)=-\frac{1}{\sqrt{\operatorname{det} g(x)}} \frac{\partial}{\partial x_{i}}\left[\sqrt{\operatorname{det} g(x)} g^{-1}(x) \frac{\partial u}{\partial x_{j}}\right]+V(x) u(x)
$$

for all $x \in R^{n}$, in the Hilbert space $L_{2}\left(R^{n}, H_{1}\right)$ with the operator potential $V(x) \in C^{1}\left(R^{n}, L\left(H_{1}\right)\right)$ and $g(x)=\left(g_{i j}(x)\right)$ is the Riemannian matrix, while $g^{-1}(x)$ is the inverse of the matrix $g(x)$.

Recently, Zayed [21] has studied the separation for the biharmonic differential operator

$$
A u(x)=\Delta \Delta u+V(x) u(x), \quad x \in R^{n},
$$

in the Hilbert space $H=L_{2}\left(R^{n}, H_{1}\right)$ with the operator potential $V(x) \in C^{1}\left(R^{n}, L\left(H_{1}\right)\right)$ and $\Delta \Delta u$ is the biharmonic differential operator, while $\Delta u=\sum_{i=1}^{n}\left(\partial^{2} u(x) / \partial x_{i}^{2}\right)$ is the Laplace operator in $R^{n}$.

Further results for separation of differential operators can be found in [22-30].

The main objective of the present paper is to study the separation for the biharmonic Laplace-Beltrami differential operator

$$
A u(x)=-\Delta \Delta u+V(x) u(x), \quad x \in R^{n},
$$

in the Hilbert space $L_{2}\left(R^{n}, H_{1}\right)$ with the operator potential $V(x) \in C^{1}\left(R^{n}, L\left(H_{1}\right)\right)$ where $\Delta \Delta u$ is the biharmonic differential operator and

$$
\Delta u=-\sum_{i, j=1}^{n} \frac{1}{\sqrt{\operatorname{det} g}} \frac{\partial}{\partial x_{i}}\left[\sqrt{\operatorname{det} g} g^{-1}(x) \frac{\partial u}{\partial x_{j}}\right],
$$

is the Laplace-Beltrami differential operator in $R^{n}$. We derive also the coercive estimate for the operator (1.9). The existence and uniqueness Theorem for the solution of the nonhomogeneous biharmonic Laplace-Beltrami differential equation

$$
A u(x)=-\Delta \Delta u(x)+V(x) u(x)=f(x),
$$

in the Hilbert space $H=L_{2}\left(R^{n}, H_{1}\right)$ is also given, where $f(x) \in H$. 


\section{Some notations}

In this section, we introduce the definitions that will be used in the subsequent section. Let $H_{1}$ be a separable Hilbert space with the norm $\|\cdot\|_{1}$ and the scalar product $\langle\cdot, \cdot\rangle_{1}$. We introduce the Hilbert space $H=L_{2}\left(R^{n}, H_{1}\right)$ of all vector functions $u(x), x \in R^{n}$ equipped with the norm

$$
\|u\|^{2}=\int_{R^{n}}\|u(x)\|_{1}^{2} \mathrm{~d} x .
$$

The symbol $\langle u, v\rangle$ where $u, v \in H$ denotes the scalar product in $H$ which is defined by

$$
\langle u, v\rangle=\int_{R^{n}}\langle u, v\rangle_{1} \mathrm{~d} x .
$$

The space of all vector functions $u(x), x \in R^{n}$, that have generalized derivatives $D^{\alpha} u(x)$, $\alpha \leq 2$ such that $u(x)$ and $D^{\alpha} u(x)$ belong to $H$ is denoted by $W_{2}^{2}\left(R^{n}, H_{1}\right)$. We say that the function $u(x) \in W_{2, l o c}^{2}\left(R^{n}, H_{1}\right)$ if for all functions $Q(x) \in C_{0}^{\infty}(R)$ the vector function $Q(x) u(x) \in W_{2}^{2}\left(R^{n}, H_{1}\right)$.

\section{Main results}

Definition 3.1. The biharmonic Laplace-Beltrami differential operator

$$
A u(x)=-\Delta \Delta u+V(x) u(x),
$$

for all $x \in R^{n}$ where $\Delta u$ is given by (10) is said to be separated in the Hilbert space $H=L_{2}\left(R^{n}, H_{1}\right)$ if the following statement holds: If $u(x) \in H \cap W_{2, l o c}^{2}\left(R^{n}, H_{1}\right)$ and $A u(x) \in H$ imply both of $\Delta \Delta u$ and $V u \in H$.

The main results in this paper have been formulated as follows:

Theorem 3.1. If the following conditions are satisfied for all $x \in R^{n}$ :

$$
\begin{aligned}
& \left\|\sum_{i, j=1}^{n} \frac{V_{0}^{1 / 2}}{\sqrt{\operatorname{det} g}} \frac{\partial}{\partial x_{i}}\left[\sqrt{\operatorname{det} g} g^{-1} \frac{\partial u}{\partial x_{j}}\right]-\sum_{i, j=1}^{n} V_{0}^{-1 / 2} \frac{\partial}{\partial x_{j}}\left[\frac{1}{2} g^{-1} V u \frac{\partial}{\partial x_{i}} \operatorname{lndet} g\right]\right\| \\
\leq & 2 \sigma_{1} n^{2}\|V u\|, \\
& \left\|V_{0}^{-1 / 2} \frac{\partial}{\partial x_{j}}\left[g^{-1} u \frac{\partial V}{\partial x_{i}}\right]\right\| \leq 2 \sigma_{2}\|V u\|,
\end{aligned}
$$

and

$$
\left\|V_{0}^{-1 / 2} \frac{\partial}{\partial x_{j}}\left[g^{-1} V \frac{\partial u}{\partial x_{i}}\right]\right\| \leq 2 \sigma_{3}\|V u\|
$$


where $V_{0}=\operatorname{ReV}$ while $\sigma_{i}(i=1,2,3)$ are positive constants satisfying the inequality $0<\sigma<\frac{1}{n^{2}}$, and $\sigma=\sum_{i=1}^{3} \sigma_{i}$. Then, the following coercive estimate is true:

$$
\|V u\|+\|\Delta \Delta u\|+\left\|\sum_{i, j=1}^{n} \frac{V_{0}^{1 / 2}}{\sqrt{\operatorname{det} g}} \frac{\partial}{\partial x_{i}}\left[\sqrt{\operatorname{det} g} g^{-1} \frac{\partial u}{\partial x_{j}}\right]\right\| \leq N\|A u\|,
$$

where

$$
N=1+2\left(1-\frac{n^{2} \sigma}{\beta}\right)^{-1}+\left(1-n^{2} \sigma \beta\right)^{-1 / 2}\left(1-\frac{n^{2} \sigma}{\beta}\right)^{-1 / 2},
$$

is a positive constant independent on $u(x)$, while $\beta$ is a positive constant satisfying the inequality $n^{2} \sigma<\beta<\frac{1}{n^{2} \sigma}$. That is, the biharmonic Laplace-Beltrami differential operator $A u(x)$ given by (1.9) is separated in the Hilbert space $H=L_{2}\left(R^{n}, H_{1}\right)$.

Proof. From the definition of the scalar product in $H$ and by integrating by parts, we obtain

$$
\left\langle\frac{\partial u}{\partial x_{i}}, v\right\rangle=-\left\langle u, \frac{\partial v}{\partial x_{i}}\right\rangle \quad \text { for all } u, v \in C_{0}^{\infty}\left(R^{n}\right)
$$

From (1.9), we get

$$
\langle A u, V u\rangle=\langle-\Delta \Delta u+V u, V u\rangle=\langle-\Delta \Delta u, V u\rangle+\langle V u, V u\rangle .
$$

On setting $\Delta u=W(x)$, we have

$$
\begin{aligned}
& \langle A u, V u\rangle=\langle-\Delta W, V u\rangle+\langle V u, V u\rangle \\
= & \left\langle\sum_{i, j=1}^{n} \frac{1}{\sqrt{\operatorname{det} g}} \frac{\partial}{\partial x_{i}}\left[\sqrt{\operatorname{det} g} g^{-1} \frac{\partial W}{\partial x_{j}}\right], V u\right\rangle+\langle V u, V u\rangle \\
= & -\sum_{i, j=1}^{n}\left\langle\sqrt{\operatorname{det} g} g^{-1} \frac{\partial W}{\partial x_{j}}, \frac{\partial}{\partial x_{i}}\left[\frac{1}{\sqrt{\operatorname{det} g}} V u\right]\right\rangle+\langle V u, V u\rangle \\
= & \frac{1}{2} \sum_{i, j=1}^{n}\left\langle g^{-1} \frac{\partial W}{\partial x_{j}}, V u \frac{\partial}{\partial x_{i}}(\ln \operatorname{det} g)\right\rangle-\sum_{i, j=1}^{n}\left\langle g^{-1} \frac{\partial W}{\partial x_{j}}, u \frac{\partial V}{\partial x_{i}}\right\rangle \\
& \quad-\sum_{i, j=1}^{n}\left\langle g^{-1} \frac{\partial W}{\partial x_{j}}, V \frac{\partial u}{\partial x_{i}}\right\rangle+\langle V u, V u\rangle \\
= & -\sum_{i, j=1}^{n}\left\langle W, \frac{\partial}{\partial x_{j}}\left[\frac{1}{2} g^{-1} V u \frac{\partial}{\partial x_{i}}(\operatorname{lndetg})\right]\right\rangle+\sum_{i, j=1}^{n}\left\langle W, \frac{\partial}{\partial x_{j}}\left[g^{-1} u \frac{\partial V}{\partial x_{i}}\right]\right\rangle \\
& \quad+\sum_{i, j=1}^{n}\left\langle W, \frac{\partial}{\partial x_{j}}\left[g^{-1} V \frac{\partial u}{\partial x_{i}}\right]\right\rangle+\langle V u, V u\rangle .
\end{aligned}
$$


On substituting (1.10) into (3.5) and equating the real parts of both sides of the resultant form, we obtain

$$
\begin{aligned}
& \operatorname{Re}\langle A u, V u\rangle \\
=\left\langle\sum_{i, j=1}^{n}\right. & \left.\frac{V_{0}^{1 / 2}}{\sqrt{\operatorname{det} g}} \frac{\partial}{\partial x_{i}}\left[\sqrt{\operatorname{det} g} g^{-1} \frac{\partial u}{\partial x_{j}}\right], \sum_{i, j=1}^{n} \frac{V_{0}^{1 / 2}}{\sqrt{\operatorname{det} g}} \frac{\partial}{\partial x_{i}}\left[\sqrt{\operatorname{det} g} g^{-1} \frac{\partial u}{\partial x_{j}}\right]\right\rangle \\
& -\operatorname{Re}\left\langle\sum_{i, j=1}^{n} \frac{V_{0}^{1 / 2}}{\sqrt{\operatorname{det} g}} \frac{\partial}{\partial x_{i}}\left[\sqrt{\operatorname{det} g} g^{-1} \frac{\partial u}{\partial x_{j}}\right], \sum_{i, j=1}^{n} \frac{V_{0}^{1 / 2}}{\sqrt{\operatorname{det} g}} \frac{\partial}{\partial x_{i}}\left[\sqrt{\operatorname{det} g} g^{-1} \frac{\partial u}{\partial x_{j}}\right]\right\rangle \\
& +\operatorname{Re}\left\langle\sum_{i, j=1}^{n} \frac{V_{0}^{1 / 2}}{\sqrt{\operatorname{det} g}} \frac{\partial}{\partial x_{i}}\left[\sqrt{\operatorname{det} g} g^{-1} \frac{\partial u}{\partial x_{j}}\right], \sum_{i, j=1}^{n} V_{0}^{-1 / 2} \frac{\partial}{\partial x_{j}}\left[\frac{1}{2} g^{-1} V u \frac{\partial}{\partial x_{i}}(\operatorname{lndet} g)\right]\right\rangle \\
& -\operatorname{Re}\left\langle\sum_{i, j=1}^{n} \frac{V_{0}^{1 / 2}}{\sqrt{\operatorname{det} g}} \frac{\partial}{\partial x_{i}}\left[\sqrt{\operatorname{det} g} g^{-1} \frac{\partial u}{\partial x_{j}}\right], \sum_{i, j=1}^{n} V_{0}^{-1 / 2} \frac{\partial}{\partial x_{j}}\left[g^{-1} u \frac{\partial V}{\partial x_{i}}\right]\right\rangle \\
& -\operatorname{Re}\left\langle\sum_{i, j=1}^{n} \frac{V_{0}^{1 / 2}}{\sqrt{\operatorname{det} g}} \frac{\partial}{\partial x_{i}}\left[\sqrt{\operatorname{det} g} g^{-1} \frac{\partial u}{\partial x_{j}}\right], \sum_{i, j=1}^{n} V_{0}^{-1 / 2} \frac{\partial}{\partial x_{j}}\left[g^{-1} V \frac{\partial u}{\partial x_{i}}\right]\right\rangle \\
& +\langle V u, V u\rangle .
\end{aligned}
$$

Since for any complex number $Z$, we have

$$
-|Z| \leq \operatorname{Re} Z \leq|Z|,
$$

then by using the Cauchy-Schwarz inequality, we get

$$
\operatorname{Re}\langle A u, V u\rangle \leq|\langle A u, V u\rangle| \leq\|A u\|\|V u\| .
$$

Further, since for any $\beta>0$ and $y_{1}, y_{2} \in R^{n}$, then with reference to [18] we have

$$
\left|y_{1}\right|\left|y_{2}\right| \leq \frac{\beta}{2}\left|y_{1}\right|^{2}+\frac{1}{2 \beta}\left|y_{2}\right|^{2}
$$

Consequently, we deduce from (3.1)-(3.3) and (3.7)-(3.9) that

$$
\begin{aligned}
& -\operatorname{Re}\left\langle K_{1}, K_{1}-\sum_{i, j=1}^{n} V_{0}^{-1 / 2} \frac{\partial}{\partial x_{j}}\left[\frac{1}{2} g^{-1} V u \frac{\partial}{\partial x_{i}}(\operatorname{lndet} g)\right]\right\rangle \\
\geq & -n^{2} \sigma_{1} \beta\left\|\sum_{i, j=1}^{n} \frac{V_{0}^{1 / 2}}{\sqrt{\operatorname{det} g}} \frac{\partial}{\partial x_{i}}\left[\sqrt{\operatorname{det} g} g^{-1} \frac{\partial u}{\partial x_{j}}\right]\right\|^{2}-\frac{n^{2} \sigma_{1}}{\beta}\|V u\|^{2}, \\
& -\operatorname{Re}\left\langle\sum_{i, j=1}^{n} \frac{V_{0}^{1 / 2}}{\sqrt{\operatorname{det} g}} \frac{\partial}{\partial x_{i}}\left[\sqrt{\operatorname{det} g} g^{-1} \frac{\partial u}{\partial x_{j}}\right], \sum_{i, j=1}^{n} V_{0}^{-1 / 2} \frac{\partial}{\partial x_{j}}\left[g^{-1} u \frac{\partial V}{\partial x_{i}}\right]\right\rangle
\end{aligned}
$$




$$
\geq-n^{2} \sigma_{2} \beta\left\|\sum_{i, j=1}^{n} \frac{V_{0}^{1 / 2}}{\sqrt{\operatorname{det} g}} \frac{\partial}{\partial x_{i}}\left[\sqrt{\operatorname{det} g} g^{-1} \frac{\partial u}{\partial x_{j}}\right]\right\|^{2}-\frac{n^{2} \sigma_{2}}{\beta}\|V u\|^{2},
$$

and

$$
\begin{aligned}
& -\operatorname{Re}\left\langle\sum_{i, j=1}^{n} \frac{V_{0}^{1 / 2}}{\sqrt{\operatorname{det} g}} \frac{\partial}{\partial x_{i}}\left[\sqrt{\operatorname{det} g} g^{-1} \frac{\partial u}{\partial x_{j}}\right], \sum_{i, j=1}^{n} V_{0}^{-1 / 2} \frac{\partial}{\partial x_{j}}\left[g^{-1} V \frac{\partial u}{\partial x_{i}}\right]\right\rangle \\
\geq & -n^{2} \sigma_{3} \beta\left\|\sum_{i, j=1}^{n} \frac{V_{0}^{1 / 2}}{\sqrt{\operatorname{det} g}} \frac{\partial}{\partial x_{i}}\left[\sqrt{\operatorname{det} g} g^{-1} \frac{\partial u}{\partial x_{j}}\right]\right\|^{2}-\frac{n^{2} \sigma_{3}}{\beta}\|V u\|^{2},
\end{aligned}
$$

On substituting (3.10)-(3.12) into (3.6) we get the inequality

$$
\left(1-\frac{n^{2} \sigma}{\beta}\right)\|V u\|^{2}+\left(1-n^{2} \sigma \beta\right)\left\|\sum_{i, j=1}^{n} \frac{V_{0}^{1 / 2}}{\sqrt{\operatorname{det} g}} \frac{\partial}{\partial x_{i}}\left[\sqrt{\operatorname{det} g} g^{-1} \frac{\partial u}{\partial x_{j}}\right]\right\|^{2} \leq\|A u\|\|V u\|,
$$

where $\sigma=\sum_{i=1}^{3} \sigma_{i}$. Choosing $n^{2} \sigma<\beta<\frac{1}{n^{2} \sigma}$, we deduce from (3.13) that

$$
\begin{aligned}
& \|V u\| \leq\left(1-\frac{n^{2} \sigma}{\beta}\right)^{-1}\|A u\| . \\
& \left\|\sum_{i, j=1}^{n} \frac{V_{0}^{1 / 2}}{\sqrt{\operatorname{det} g}} \frac{\partial}{\partial x_{i}}\left[\sqrt{\operatorname{det} g} g^{-1} \frac{\partial u}{\partial x_{j}}\right]\right\| \leq\left(1-n^{2} \beta \sigma\right)^{-1 / 2}\left(1-\frac{n^{2} \sigma}{\beta}\right)^{-1 / 2}\|A u\| .
\end{aligned}
$$

Since $A u=-\Delta \Delta u+V u$, then we get

$$
\|\Delta \Delta u\| \leq\|A u\|+\|V u\| \leq\left\{1+\left(1-\frac{n^{2} \sigma}{\beta}\right)^{-1}\right\}\|A u\| .
$$

From the inequalities (3.14)-(3.16) we arrive at the coercive estimate (3.4). Hence, the proof of Theorem 3.1 is completed.

Theorem 3.2. If the biharmonic Laplace-Beltrami differential operator $A u(x)$ given by (1.9) is separated in the Hilbert space $H=L_{2}\left(R^{n}, H_{1}\right)$ and if there are positive functions $t(x), \psi(x) \in$ $C^{1}\left(R^{n}\right)$ such that the following conditions are true:

$$
\begin{aligned}
& \left\|t^{1 / 2} \psi^{1 / 2} g^{-1 / 2} \frac{\partial u}{\partial x_{j}}\right\| \leq 2 \sqrt{\rho_{1}}\left\|t^{1 / 2} \psi^{1 / 2} V_{0}^{1 / 2} u\right\|, \\
& \left\|g^{-1 / 2} \psi^{-1} \frac{\partial \psi}{\partial x_{i}} V_{0}^{-1 / 2}\right\| \leq 2 \sqrt{\rho_{2}}, \\
& \left\|g^{-1 / 2} t^{-1} \frac{\partial t}{\partial x_{j}} V_{0}^{-1 / 2}\right\| \leq 2 \sqrt{\rho_{3}},
\end{aligned}
$$




$$
\left\|\frac{1}{2} g^{-1 / 2} V_{0}^{-1 / 2} \frac{\partial}{\partial x_{j}}(\ln \operatorname{det} g)\right\| \leq 2 \sqrt{\rho_{4}}
$$

and

$$
\left\|t^{1 / 2} \psi^{1 / 2} g^{-1 / 2} \frac{\partial W}{\partial x_{i}}\right\| \leq\left\|t^{1 / 2} \psi^{1 / 2} g^{-1 / 2} \frac{\partial \psi}{\partial x_{i}}\right\|
$$

where $\rho_{i}(i=1-4)$ are positive constants satisfying $0<\rho<\frac{\beta}{2 n^{2}}$ and $\rho=\sum_{i=1}^{4} \rho_{i}$ while $V_{0}=\operatorname{ReV}$. Then, the non-homogeneous biharmonic Laplace-Beltrami differential equation

$$
A u=-\Delta \Delta u+V(x) u(x)=f(x),
$$

has a unique solution in the Hilbert space $H$, where $f(x) \in H$.

Proof. First, we prove that the homogeneous biharmonic Laplace-Beltrami differential equation

$$
A u(x)=-\Delta \Delta u(x)+V(x) u(x)=0,
$$

has only the zero solution $u(x)=0$ for all $x \in R^{n}$. To this end, we assume that $t(x)$ and $\psi(x) \in C^{1}\left(R^{n}\right)$ are postive functions. Thus, on setting $\Delta u(x)=W(x)$, we have

$$
\begin{aligned}
\langle V u, t \psi u\rangle= & \langle\Delta \Delta u, t \psi u\rangle=\langle\Delta W, t \psi u\rangle \\
= & -\left\langle\sum_{i, j=1}^{n} \frac{1}{\sqrt{\operatorname{det} g}} \frac{\partial}{\partial x_{j}}\left[\sqrt{\operatorname{det} g} g^{-1} \frac{\partial W}{\partial x_{i}}\right], t \psi u\right\rangle \\
= & \sum_{i, j=1}^{n}\left\langle\sqrt{\operatorname{det} g} g^{-1} \frac{\partial W}{\partial x_{i}}, \frac{\partial}{\partial x_{j}}\left(\frac{1}{\sqrt{\operatorname{det} g}} t \psi u\right)\right\rangle \\
= & \sum_{i, j=1}^{n}\left\langle\sqrt{\operatorname{det} g} g^{-1} \frac{\partial W}{\partial x_{i}}, \frac{1}{\sqrt{\operatorname{det} g}} \frac{\partial}{\partial x_{j}}(t \psi u)\right\rangle \\
& \quad+\sum_{i, j=1}^{n}\left\langle\sqrt{\operatorname{det} g} g^{-1} \frac{\partial W}{\partial x_{i}}, t \psi u \frac{\partial}{\partial x_{j}}\left(\frac{1}{\sqrt{\operatorname{det} g}}\right)\right\rangle \\
= & \sum_{i, j=1}^{n}\left\langle\frac{\partial W}{\partial x_{i}}, g^{-1} \frac{\partial}{\partial x_{j}}(t \psi u)\right\rangle-\frac{1}{2} \sum_{i, j=1}^{n}\left\langle\frac{\partial W}{\partial x_{i}}, g^{-1} t \psi u \frac{\partial}{\partial x_{j}}(\operatorname{lndet} g)\right\rangle \\
= & \sum_{i, j=1}^{n}\left\langle\frac{\partial W}{\partial x_{i}}, g^{-1} t \psi \frac{\partial u}{\partial x_{j}}\right\rangle+\sum_{i, j=1}^{n}\left\langle\frac{\partial W}{\partial x_{i}}, g^{-1} t u \frac{\partial \psi}{\partial x_{j}}\right\rangle \\
& \quad+\sum_{i, j=1}^{n}\left\langle\frac{\partial W}{\partial x_{i}}, g^{-1} \psi u \frac{\partial t}{\partial x_{j}}\right\rangle-\frac{1}{2} \sum_{i, j=1}^{n}\left\langle\frac{\partial W}{\partial x_{i}}, g^{-1} t \psi u \frac{\partial}{\partial x_{j}}(\operatorname{lndet} g)\right\rangle .
\end{aligned}
$$

Equating the real parts of both sides of (3.24), we get

$$
\left\langle V_{0} u, t \psi u\right\rangle=\left\langle t^{1 / 2} \psi^{1 / 2} V_{0}^{1 / 2} u, t^{1 / 2} \psi^{1 / 2} V_{0}^{1 / 2} u\right\rangle
$$




$$
\begin{aligned}
=\sum_{i, j=1}^{n} & \operatorname{Re}\left\langle t^{1 / 2} \psi^{1 / 2} g^{-1 / 2} \frac{\partial W}{\partial x_{i}}, t^{1 / 2} \psi^{1 / 2} g^{-1 / 2} \frac{\partial u}{\partial x_{j}}\right\rangle \\
& +\sum_{i, j=1}^{n} \operatorname{Re}\left\langle t^{1 / 2} \psi^{1 / 2} g^{-1 / 2} \frac{\partial W}{\partial x_{i}}, t^{1 / 2} \psi^{1 / 2}\left[g^{-1 / 2} \psi^{-1} \frac{\partial \psi}{\partial x_{j}} V_{0}^{-1 / 2}\right] V_{0}^{1 / 2} u\right\rangle \\
& +\sum_{i, j=1}^{n} \operatorname{Re}\left\langle t^{1 / 2} \psi^{1 / 2} g^{-1 / 2} \frac{\partial W}{\partial x_{i}}, t^{1 / 2} \psi^{1 / 2}\left[g^{-1 / 2} t^{-1} \frac{\partial t}{\partial x_{j}} V_{0}^{-1 / 2}\right] V_{0}^{1 / 2} u\right\rangle \\
& -\sum_{i, j=1}^{n} \operatorname{Re}\left\langle t^{1 / 2} \psi^{1 / 2} g^{-1 / 2} \frac{\partial W}{\partial x_{i}}, t^{1 / 2} \psi^{1 / 2}\left[\frac{1}{2} g^{-1 / 2} V_{0}^{-1 / 2} \frac{\partial}{\partial x_{j}}(\operatorname{lndet} g)\right] V_{0}^{1 / 2} u\right\rangle .
\end{aligned}
$$

With the aid of (3.7)-(3.9) together with the inequalities (3.17)-(3.21), we deduce that (3.25) takes the form

$$
\begin{aligned}
&\left\|t^{1 / 2} \psi^{1 / 2} V_{0}^{1 / 2} u\right\|^{2} \leq \frac{n \beta}{2} \sum_{i=1}^{n}\left\|t^{1 / 2} \psi^{1 / 2} g^{-1 / 2} \frac{\partial W}{\partial x_{i}}\right\|^{2}+\frac{2 n^{2} \rho_{1}}{\beta}\left\|t^{1 / 2} \psi^{1 / 2} V_{0}^{1 / 2} u\right\|^{2} \\
&+ \frac{n \beta}{2} \sum_{i=1}^{n}\left\|t^{1 / 2} \psi^{1 / 2} g^{-1 / 2} \frac{\partial W}{\partial x_{i}}\right\|^{2}+\frac{2 n^{2} \rho_{2}}{\beta}\left\|t^{1 / 2} \psi^{1 / 2} V_{0}^{1 / 2} u\right\|^{2} \\
&+\frac{n \beta}{2} \sum_{i=1}^{n}\left\|t^{1 / 2} \psi^{1 / 2} g^{-1 / 2} \frac{\partial W}{\partial x_{i}}\right\|^{2}+\frac{2 n^{2} \rho_{3}}{\beta}\left\|t^{1 / 2} \psi^{1 / 2} V_{0}^{1 / 2} u\right\|^{2} \\
&+\frac{n \beta}{2} \sum_{i=1}^{n}\left\|t^{1 / 2} \psi^{1 / 2} g^{-1 / 2} \frac{\partial W}{\partial x_{i}}\right\|^{2}+\frac{2 n^{2} \rho_{4}}{\beta}\left\|t^{1 / 2} \psi^{1 / 2} V_{0}^{1 / 2} u\right\|^{2} .
\end{aligned}
$$

Consequently, if we put $\rho=\sum_{i=1}^{4} \rho_{i}$, then (3.26) becomes in the form

$$
\left(1-\frac{2 n^{2} \rho}{\beta}\right)\left\|t^{1 / 2} \psi^{1 / 2} V_{0}^{1 / 2} u\right\|^{2} \leq 2 n \beta \sum_{i=1}^{n}\left\|t^{1 / 2} \psi^{1 / 2} g^{-1 / 2} \frac{\partial \psi}{\partial x_{i}}\right\|^{2},
$$

By choosing $\psi(x)=1$ for all $x \in R^{n}$, then if $0<\rho<\frac{\beta}{2 n^{2}}$ we see that (3.27) becomes in the form

$$
0<\left(1-\frac{2 n^{2} \rho}{\beta}\right)\left\|t^{1 / 2} V_{0}^{1 / 2} u\right\|^{2} \leq 0
$$

From (2.1) and (3.28) we obtain

$$
0<\left(1-\frac{2 n^{2} \rho}{\beta}\right) \int_{R^{n}}\left\|t^{1 / 2} V_{0}^{1 / 2} u\right\|_{1}^{2} d x \leq 0 .
$$

Now, the inequality (3.29) holds only for $u(x)=0$. This prove that $u(x)=0$ is the only solution of the homogeneous biharmonic Laplace-Beltrami differential equation (3.23). Furthermore, it is easy to check that the linear manifold

$$
L=\left\{f: A u(x)=f(x), \quad \text { for all } f \in C_{0}^{\infty}\left(R^{n}\right)\right\}
$$


is dense in $H$. So, we can construct a sequence of vector functions $\left\{y_{r}\right\} \in C_{0}^{\infty}\left(R^{n}\right)$ where $\left\|y_{r}\right\| \neq 0$ for all $r$ such that $\left\|A y_{r}-f\right\| \rightarrow 0$ as $r \rightarrow \infty$ for all $f \in H$. On using the coercive estimate (3.4), we have

$$
\begin{aligned}
& \quad\left\|V\left(y_{p}-y_{r}\right)\right\|+\left\|\Delta \Delta\left(y_{p}-y_{r}\right)\right\|+\left\|\sum_{i, j=1}^{n} \frac{V_{0}^{1 / 2}}{\sqrt{\operatorname{det} g}} \frac{\partial}{\partial x_{i}}\left[\sqrt{\operatorname{det} g} g^{-1} \frac{\partial\left(y_{p}-y_{r}\right)}{\partial x_{j}}\right]\right\| \\
& \leq N\left\|A\left(y_{p}-y_{r}\right)\right\|,
\end{aligned}
$$

where $y_{p}-y_{r}=u$ and $p, r=1,2, \ldots$ As $p, r \rightarrow \infty$ we see from (3.30) that the sequences $\left\{V y_{r}\right\}$, $\left\{\Delta \Delta y_{r}\right\}$ and $\left\{\sum_{i, j=1}^{n} \frac{V_{0}^{1 / 2}}{\sqrt{\operatorname{det} g}} \frac{\partial}{\partial x_{i}}\left[\sqrt{\operatorname{det} g} g^{-1} \frac{\partial y_{r}}{\partial x_{j}}\right]\right\}$ are Cauchy sequences in the Hilbert space $H$ and then they are convergent. Therefore, there exists real functions $\mu_{0}(x), \mu_{1}(x)$ and $\mu_{2}(x)$ in $H$ such that

$$
\left\|V y_{r}-\mu_{0}\right\| \rightarrow 0, \quad\left\|\Delta \Delta y_{r}-\mu_{1}\right\| \rightarrow 0
$$

and

$$
\left\|\sum_{i, j=1}^{n} \frac{V_{0}^{1 / 2}}{\sqrt{\operatorname{det} g}} \frac{\partial}{\partial x_{i}}\left[\sqrt{\operatorname{det} g} g^{-1} \frac{\partial y_{r}}{\partial x_{j}}\right]-\mu_{2}\right\| \rightarrow 0 .
$$

Hence these sequences are bounded in $H$. This implies that as $r \rightarrow \infty$, we have

$$
y_{r} \rightarrow V^{-1} \mu_{0}=y, \quad \Delta \Delta y_{r} \rightarrow \Delta \Delta y,
$$

and

$$
\sum_{i, j=1}^{n} \frac{V_{0}^{1 / 2}}{\sqrt{\operatorname{det} g}} \frac{\partial}{\partial x_{i}}\left[\sqrt{\operatorname{det} g} g^{-1} \frac{\partial y_{r}}{\partial x_{j}}\right] \rightarrow \sum_{i, j=1}^{n} \frac{V_{0}^{1 / 2}}{\sqrt{\operatorname{det} g}} \frac{\partial}{\partial x_{i}}\left[\sqrt{\operatorname{det} g} g^{-1} \frac{\partial y}{\partial x_{j}}\right] .
$$

Hence for a given function $f \in H$ there exists $y \in H \cap W_{2, l o c}^{2}\left(R^{n}, H_{1}\right)$ such that $A y=f$. Suppose that $\widetilde{y}$ is another solution of the non-homogeneous biharmonic Laplace-Beltrami differential equation $A y=f$, then we get $A(y-\widetilde{y})=0$. But $A u=0$ has only the zero solution $u=0$. Then $y=\widetilde{y}$ and the uniqueness is proved. Hence, the proof of Theorem 3.2 is completed.

\section{Conclusions}

The biharmonic Laplace-Beltrami differential operator (1.9) has been investigated using the separation method in the Hilbert space $H$ with the norm (2.1) and the scalar product (2.2). In this paper we have two interesting results. The first result is Theorem 3.1, which proves that the operator (1.9) is separated in the Hilbert space $H$. The second result is Theorem 3.2, which shows that the non-homogeneous biharmonic Laplace-Beltrami differential equation (3.22) has a unique solution in the Hilbert space $H$. 


\section{Acknowledgement}

The author wishes to thank the referees for their comments on the paper.

\section{References}

[1] Everitt W. N. and Giertz M., Some properties of the domains of certain differential operators. Proc. London Math. Soc., 23 (1971), 301-324.

[2] Everitt W. N. and Giertz M., Some inequalities associated with certain differential operators. Math. Z., 126 (1972), 308-326.

[3] Everitt W. N. and Giertz M., On some properties of the powers of a familly self-adjoint differential expressions. Proc. London Math. Soc., 24 (1972), 149-170.

[4] Everitt W. N. and Giertz M., Inequalities and separation for Schrodingertype operators in $L_{2}\left(R^{n}\right)$. Proc. Roy. Soc. Edin., 79A (1977), 257-265.

[5] Biomatov K. Kh., Coercive estimates and separation for second order elliptic differential equations. Soviet Math. Dok1. 38 (1989). English trans1. in American Math. Soc. (1989), 157160.

[6] Otelbaev M., On the separation of elliptic operator. Dok1. Acad. Nauk SSSR Russian, 234 (1977), 540-543.

[7] Zettle A., Separation for differential operators and the $L_{p}$ spaces. Proc. Amer. Math. Soc., 55 (1976), 44-46.

[8] Mohamed A. S., Separation for Schrodinger operator with matrix potential. Dok1. Acad. Nauk Tajkctan Russian ,35 (1992), 156-159.

[9] Mohamed A. S. and El-Gendi B. A., Separation for ordniary differential equation with matrix coefficient. Collect. Math., 48 (1997), 243-252.

[10] Mohamed A. S., Existence and uniqueness of the solution, separation for certain second order elliptic differential equation. Applicable Analysis, 76 (2000), 179-185.

[11] Mohamed A. S. and Atia H. A., Separation of the Sturm-Liouville differential operator with an operator potential. Applied Mathematics and Computation, 156 (2004), 387-394.

[12] Mohamed A. S. and Atia H. A., Separation of the Schrodinger operator with an operator potential in the Hilbert spaces. Applicable Analysis, 84 (2005), 103-110.

[13] Mohamed A. S. and Atia H. A., Separation of the general second order elliptic differential operator with an operator potential in the weighted Hilbert spaces. Applied Mathematics and Computation, 162 (2005), 155-163.

[14] Bergbaev A., Smooth solution of non-linear differential equation with matrix potential. the VII Scientific Conference of Mathematics and Mechanics AlmaAta, Russian, (1989).

[15] Brown R. C., Separation and disconjugacy. J. Inequal. Pure Appl. Math., 4 (2003) Art. 56.

[16] Brown R. C. and Hinton D. B., Two separation criteria for second order ordinary or partial differential operators. Math. Bohemica, 124 (1999), 273-292.

[17] Brown R. C. and Hinton D. B. and Shaw M. F., Some separation criteria and inequalities associated with linear second order differential operators, in function spaces and applications. Narosa Publishing House, New Delhi, (2000), 7-35.

[18] Zayed E. M. E., Mohamed A. S. and Atia H. A., Separation for Schrodinger type operators with operator potential in Banach spaces. Applicable Analysis, 84 (2005), 211-220. 
[19] Zayed E. M. E., Mohamed A. S. and Atia H. A., On the separation of elliptic differential operators with operator potential in weighted Hilbert spaces. Panamerican Mathematical Journal, 15 (2005), 39-47.

[20] Zayed E. M. E., Mohamed A. S. and Atia H. A., Inequalities and separation for the Laplace Beltrami differential operator in Hilbert spaces. J. Math. Anal. Appl., 336 (2007), 81-92.

[21] Zayed E. M. E., Separation for the biharmonic differential operator in the Hilbert space associated with the existence and uniqueness Theorem. J. Math. Anal. Appl. 337 (2008), 659-666.

[22] Atia H. A. and Mahmoud R. A., Separation of the two-dimensional Laplace operator by disconjugacy property. Panamerican Mathematical Journal, 20 (2009) 93-103.

[23] Atia H. A., Separation of the Grushin differential operator in the weighted Hilbert spaces. Lobac hevskii J. Math., 32 (2011) 180-188.

[24] Atia H. A., Separation problem for the Grushin differential operator in Banach spaces. Carpathian J. Math., 30 (2014) 31-37.

[25] Atia H. A., Ramzi S. A. and Ahmed R., Separation of biharmonic differential operators on Riemannian monifolds. Forum Math., 26 (2014) 953-966.

[26] Ospanov K. N. and Akhmetkaliyeva R. D., Separation and the existence theorem for second order nonlinear differential equation. Electronic J. Qualitative Theory of Differential Equations, 66 (2012) 1-12.

[27] Zayed E. M. E. and Omran S. A., Separation for triple Harmonic differential operator in Hilbert space. Int. J. Math. Combinatorics, 4 (2010), 13-23.

[28] Zayed E. M. E. and Omran S. A., Separation of the Tricomi differential operator in Hilbert space with application to the existence and uniqueness theorem. Int. J. Contemp. Math. Sci., 6 (2011), 353-364.

[29] Zayed E. M. E., Separation for a biharmonic higher-order ordinary differential equation in a Hilbert space associated with an existence and uniqueness theorem. Commu. Appl. Nonlinear Anal., 22 (2015) 25-33.

[30] Zayed E. M. E., On the separation for a biharmonic general elliptic differential operator in a weighted Hilbert space with its applications to an existence and uniqueness theorem. Advances in Nonlinear Variational Inequalities, 18 (2015) 77-90. 